\title{
Global Weak Solutions of the Navier-Stokes System with Nonzero Boundary Conditions
}

\author{
By \\ R. FArwig, H. Kozono and H. SoHr \\ (Technische Universität Darmstadt, Germany, Tôhoku University, \\ Japan and Universität Paderborn, Germany) \\ In memory of our colleague Prof. Tetsuro Miyakawa
}

\begin{abstract}
Consider the Navier-Stokes equations in a smooth bounded domain $\Omega \subset \boldsymbol{R}^{3}$ and a time interval $[0, T), 0<T \leq \infty$. It is well-known that there exists at least one global weak solution $u$ with vanishing boundary values $\left.u\right|_{\partial \Omega}=0$ for any given initial value $u_{0} \in L_{\sigma}^{2}(\Omega)$, external force $f=\operatorname{div} F, F \in L^{2}\left(0, T ; L^{2}(\Omega)\right)$, and satisfying the strong energy inequality. Our aim is to extend this existence result to a much larger class of global in time "Leray-Hopf type" weak solutions $u$ with nonzero boundary values $\left.u\right|_{\partial \Omega}=g \in W^{1 / 2,2}(\partial \Omega)$. As for usual weak solutions we do not need any smallness condition on $g$; indeed, our generalized weak solutions $u$ exist globally in time. The solutions will satisfy an energy estimate with exponentially increasing terms in time, but for simply connected domains the energy increases at most linearly in time.

Key Words and Phrases. Navier-Stokes equations, Weak solution, Nonhomogeneous boundary values, Strong energy inequality.

2000 Mathematics Subject Classification Numbers. 76D05, 35Q30, 35J65.
\end{abstract}

\section{Introduction and main results}

Throughout this paper $\Omega \subset \boldsymbol{R}^{3}$ denotes a bounded domain with boundary $\partial \Omega$ of class $C^{1,1}$ and $[0, T), 0<T \leq \infty$, is a given time interval. We are interested in the Navier-Stokes system

$$
\begin{aligned}
u_{t}-\Delta u+u \cdot \nabla u+\nabla p & =f, & \operatorname{div} u & =0 \\
\left.u\right|_{\partial \Omega} & =g, & u(0) & =u_{0}
\end{aligned}
$$

in $[0, T) \times \Omega$ where the initial value $u_{0}$, the boundary values $g$ and the external force $f$ satisfy the properties

$$
u_{0} \in L^{2}(\Omega), \quad g \in W^{1 / 2,2}(\partial \Omega), \quad f=\operatorname{div} F, \quad F \in L^{2}\left(0, T ; L^{2}(\Omega)\right)
$$

and the compatibility conditions

$$
\operatorname{div} u_{0}=0 \text { in } \Omega,\left.\quad N \cdot u_{0}\right|_{\partial \Omega}=N \cdot g .
$$


Here $N=N(x)$ denotes the exterior normal vector at $x \in \partial \Omega$, so that (1.3) yields the flux condition $\int_{\partial \Omega} N \cdot g d \sigma=0$.

Before discussing (1.1) with $g \neq 0$ let us recall some classical results in the case $g=0$.

Definition 1.1. Let $u_{0} \in L_{\sigma}^{2}(\Omega)$ and $f=\operatorname{div} F, F \in L^{2}\left(0, T ; L^{2}(\Omega)\right)$. Then a vector field

$$
u \in L^{\infty}\left(0, T ; L_{\sigma}^{2}(\Omega)\right) \cap L^{2}\left(0, T ; W_{0}^{1,2}(\Omega)\right)
$$

is called a weak (Leray-Hopf) solution of $(1.1)$ in $[0, T) \times \Omega$ with data $u_{0}, g=0$, $f$ if

$$
-\left\langle u, w_{t}\right\rangle_{\Omega, T}+\langle\nabla u, \nabla w\rangle_{\Omega, T}-\langle u u, \nabla w\rangle_{\Omega, T}=\left\langle u_{0}, w(0)\right\rangle_{\Omega}-\langle F, \nabla w\rangle_{\Omega, T}
$$

is satisfied for each test function $w \in C_{0}^{\infty}\left([0, T) ; C_{0, \sigma}^{\infty}(\Omega)\right)$, and $u$ satisfies the energy inequality

$$
\frac{1}{2}\|u(t)\|_{2}^{2}+\int_{0}^{t}\|\nabla u\|_{2}^{2} d \tau \leq \frac{1}{2}\left\|u_{0}\right\|_{2}^{2}-\int_{0}^{t}\langle F, \nabla u\rangle_{\Omega} d \tau
$$

for $0 \leq t<T$.

In this definition $\langle\cdot, \cdot\rangle_{\Omega}$ denotes the pairing of vector fields in $\Omega$, and $\langle\cdot, \cdot\rangle_{\Omega, T}$ means the corresponding pairing in $[0, T) \times \Omega$. Given a vector field $u$ on $\Omega$, let $u \cdot \nabla u=(u \cdot \nabla) u=u_{1} D_{1} u+u_{2} D_{2} u+u_{3} D_{3} u$ where $D_{j}=\partial / \partial x_{j}, j=1,2,3$. For a matrix field $F=\left(F_{i j}\right)_{i, j=1}^{3}$ let $f=\operatorname{div} F=\left(D_{1} F_{1 j}+D_{2} F_{2 j}+D_{3} F_{3 j}\right)_{j=1}^{3}$ so that when $\operatorname{div} u=0$ and $u u=\left(u_{i} u_{j}\right)_{i, j=1}^{3}$, we have $u \cdot \nabla u=\operatorname{div}(u u)$. With $C_{0, \sigma}^{\infty}(\Omega)=$ $\left\{v \in C_{0}^{\infty}(\Omega): \operatorname{div} v=0\right\}$ we define $L_{\sigma}^{2}(\Omega)=\overline{C_{0, \sigma}^{\infty}(\Omega)}\|\cdot\|_{2}$ where $\|\cdot\|_{q}$ denotes the norm of the Lebesgue space $L^{q}(\Omega), 1 \leq q \leq \infty$. For vector fields $u$, it will be convenient to define the $L^{q}$-norm by the $L^{q}$-norm of the scalar function $|u|$, where $|\cdot|$ denotes the Euclidean norm in $\boldsymbol{R}^{3}$. Further, $W^{k, q}(\Omega), k \in \boldsymbol{N}_{0}$, $1 \leq q<\infty$, and $W_{0}^{k, q}(\Omega)=\overline{C_{0}^{\infty}(\Omega)}\|\cdot\|_{W^{k, q}(\Omega)}$ denote the usual Sobolev spaces. Finally we need the Bochner spaces $L^{s}\left(0, T ; L^{q}(\Omega)\right), 1<s, q<\infty$, with norm

$$
\|\cdot\|_{L^{s}\left(0, T ; L^{q}(\Omega)\right)}=\|\cdot\|_{q, s ; T}=\left(\int_{0}^{T}\|\cdot\|_{q}^{s} d \tau\right)^{1 / s}
$$

and correspondingly the spaces $L^{\infty}\left(0, T ; L^{2}(\Omega)\right), \quad L_{\mathrm{loc}}^{\infty}\left([0, T) ; L^{2}(\Omega)\right)$, and $L^{2}\left(0, T ; W_{0}^{1,2}(\Omega)\right)$. The trace space of functions in $W^{1, q}(\Omega), 1<q<\infty$, is denoted by $W^{1-1 / q, q}(\partial \Omega)$, its dual space by $W^{-1 / q^{\prime}, q^{\prime}}(\partial \Omega), q^{\prime}=q /(q-1)$. The surface measure on $\partial \Omega$ is called $d \sigma$.

As is well-known there always exists at least one weak solution $u$ in the sense of Definition 1.1 of the Navier-Stokes system (1.1) with $g=0$. We 
may assume without loss of generality (after modifying $u$ on a null set in $(0, T)$ ) that $u:[0, T) \rightarrow L_{\sigma}^{2}(\Omega)$ is weakly continuous. Thus $\left.u\right|_{t=0}=u_{0}$ is well-defined. Further, there exists a distribution $p$ in $(0, T) \times \Omega$ such that

$$
u_{t}-\Delta u+u \cdot \nabla u+\nabla p=f \quad \text { in }(0, T) \times \Omega
$$

in the sense of distributions. Finally, from (1.5) and the Cauchy-Schwarz inequality we obtain the energy estimate

$$
\|u(t)\|_{2}^{2}+\int_{0}^{t}\|\nabla u\|_{2}^{2} d \tau \leq\left\|u_{0}\right\|_{2}^{2}+\int_{0}^{t}\|F\|_{2}^{2} d \tau, \quad 0 \leq t<T .
$$

There are only very few results on the three-dimensional case of nonhomogeneous boundary values $\left.u\right|_{\partial \Omega}=g$. We refer to [10] for $L^{q}$-results with small data, [7] for the existence of local in time strong solutions and [15] for (time-dependent) boundary data of fractional regularity $W^{3 / 4,2}(\partial \Omega)$. A generalization of the present results to global weak solutions with time-dependent boundary data will be considered in a forthcoming paper [5]; there we have to assume that $g \in L^{4}\left(0, T ; W^{-1 / 4,4}(\partial \Omega)\right) \cap L^{s}\left(0, T ; W^{-1 / q, q}(\partial \Omega)\right)$ where $s, q$ are Serrin exponents satisfying $2 / s+3 / q=1$. Another approach to the NavierStokes system with nonvanishing Dirichlet boundary data in the context of socalled very weak solutions has been analyzed in $[1,2,3,4]$.

In this paper, our aim is to construct for arbitrarily large boundary values $g \in W^{1 / 2,2}(\partial \Omega)$ and arbitrary external forces $f=\operatorname{div} F, F \in L^{2}\left(0, T ; L^{2}(\Omega)\right)$ a weak solution $u$ on $[0, T)$ satisfying an appropriate energy inequality. The idea is to reduce the problem to the case of vanishing boundary values and a Navier-Stokes system containing additionally linear perturbation terms. We note that the concept of perturbed Navier-Stokes equations as in (1.10), (1.12) is also used in [6] to find the optimal initial value condition for the existence of local strong solutions.

First we have to find a suitable extension $E \in W^{1,2}(\Omega)$ of the given boundary data

$$
g \in W^{1 / 2,2}(\partial \Omega) \quad \text { with } \int_{\partial \Omega} N \cdot g d \sigma=0
$$

E.g., $E$ is the uniquely determined weak solution of the stationary Stokes system

$$
-\Delta E+\nabla \tilde{p}=f_{0}=\operatorname{div} F_{0}, \quad \operatorname{div} E=0,\left.\quad E\right|_{\partial \Omega}=g
$$

with data $g, f_{0}=\operatorname{div} F_{0}$ where $F_{0} \in L^{2}(\Omega)$, and pressure $\tilde{p} \in L^{2}(\Omega)$ satisfying the estimate

$$
\|E\|_{W^{1,2}(\Omega)} \leq c\left(\left\|F_{0}\right\|_{2}+\|g\|_{W^{1 / 2,2}(\partial \Omega)}\right)
$$


with a constant $c=c(\Omega)>0$, see [8, IV. Theorem 1.1]. In particular, if $F_{0}=0$, then the map $g \mapsto E$ is a well-defined linear bounded extension operator from $W^{1 / 2,2}(\partial \Omega)$ to $W^{1,2}(\Omega)$. However, we will see that it is reasonable to consider also the inhomogeneous Stokes system (1.8) with $f_{0} \neq 0$.

Now, setting $v=u-E$ and $q=p-\tilde{p}$ we can write (1.1) in the form

$$
\begin{aligned}
v_{t}-\Delta v+(v+E) \cdot \nabla(v+E)+\nabla q & =f-f_{0}, & \operatorname{div} v & =0 \\
\left.v\right|_{\partial \Omega} & =0, & v(0) & =v_{0}
\end{aligned}
$$

where $v_{0}=u_{0}-E$. Since

$$
(v+E) \cdot \nabla(v+E)=v \cdot \nabla v+(v \cdot \nabla E+E \cdot \nabla v)+E \cdot \nabla E,
$$

the system (1.10) may be considered as a perturbation of the usual NavierStokes system with zero boundary conditions, using the perturbation terms $E \cdot \nabla E$ and $v \cdot \nabla E+E \cdot \nabla v$. Moreover, formally testing (1.10) with a weak solution $v$ itself and noting the identity $\langle(v+E) \cdot \nabla v, v\rangle_{\Omega}=(1 / 2) \int_{\Omega}(v+E) \cdot \nabla|v|^{2} d x=0$, we get the energy inequality (1.13) below. Since $v:(0, T] \rightarrow L_{\sigma}^{2}(\Omega)$ is weakly continuous (as in the case above), we conclude that $\left.v\right|_{t=0}=v_{0}$ is well-defined, $v_{0} \in L_{\sigma}^{2}(\Omega)$ and $\operatorname{div} u_{0}=0,\left.N \cdot u_{0}\right|_{\partial \Omega}=N \cdot g$ as in (1.3).

These considerations lead to the following definition:

Definition 1.2 (Navier-Stokes system with $\left.u\right|_{\partial \Omega}=g$ ). Let $u_{0} \in L^{2}(\Omega), g \in$ $W^{1 / 2,2}(\partial \Omega)$ satisfy the compatibility conditions $\operatorname{div} u_{0}=0$ in $\Omega,\left.N \cdot u_{0}\right|_{\partial \Omega}=N \cdot g$, cf. (1.3), let $f=\operatorname{div} F, F \in L^{2}\left(0, T ; L^{2}(\Omega)\right)$, be given, and let $E \in W^{1,2}(\Omega)$ be the weak solution of the Stokes system (1.8) with data $g, f_{0}=\operatorname{div} F_{0}$ where $F_{0} \in L^{2}(\Omega)$, satisfying the a priori estimate (1.9). Then a vector field $u=v+E$ is called a weak (Leray-Hopf) solution of the general Navier-Stokes system (1.1) in $[0, T) \times \Omega$ with data $u_{0}, g$ and $f$ if $v$ satisfies the following conditions:

$$
v \in L_{\mathrm{loc}}^{\infty}\left([0, T) ; L_{\sigma}^{2}(\Omega)\right) \cap L_{\mathrm{loc}}^{2}\left([0, T) ; W_{0}^{1,2}(\Omega)\right),
$$

(ii) for all $w \in C_{0}^{\infty}\left([0, T) ; C_{0, \sigma}^{\infty}(\Omega)\right)$

$$
\begin{gathered}
-\left\langle v, w_{t}\right\rangle_{\Omega, T}+\langle\nabla v, \nabla w\rangle_{\Omega, T}-\langle(v+E)(v+E), \nabla w\rangle_{\Omega, T} \\
=\left\langle v_{0}, w(0)\right\rangle_{\Omega}-\left\langle F-F_{0}, \nabla w\right\rangle_{\Omega, T}
\end{gathered}
$$

where $v_{0}=u_{0}-E$,

(iii) the energy inequality

$$
\frac{1}{2}\|v(t)\|_{2}^{2}+\int_{0}^{t}\|\nabla v\|_{2}^{2} d \tau \leq \frac{1}{2}\left\|v_{0}\right\|_{2}^{2}-\int_{0}^{t}\left\langle F-F_{0}-(v+E) E, \nabla v\right\rangle_{\Omega} d \tau
$$

for all $0 \leq t<T$. 
The vector field $v$ is called a weak solution of the perturbed Navier-Stokes system (1.10) with initial datum $v(0)=v_{0}=u_{0}-E$.

Now our main result reads as follows:

Theorem 1.3. Let $u_{0} \in L^{2}(\Omega)$ and $g \in W^{1 / 2,2}(\partial \Omega)$ satisfy the compatibility conditions (1.3), let $f=\operatorname{div} F$ with $F \in L^{2}\left(0, T ; L^{2}(\Omega)\right)$ and $f_{0}=\operatorname{div} F_{0}$ with $F_{0} \in L^{2}(\Omega)$, and let $E \in W^{1,2}(\Omega)$ satisfy (1.8), (1.9). Then there exists at least one weak solution $u$ of the Navier-Stokes system (1.1) with data $u_{0}, g, f$ in $[0, T) \times \Omega$ in the sense of Definition 1.2, i.e., $v=u-E$ is a weak solution of the perturbed Navier-Stokes system (1.10) with $v_{0}=u_{0}-E$. This solution u satisfies the strong energy inequality

$$
\begin{aligned}
& \frac{1}{2}\|u(t)-E\|_{2}^{2}+\int_{s}^{t}\|\nabla(u-E)\|_{2}^{2} d \tau \\
& \quad \leq \frac{1}{2}\|u(s)-E\|_{2}^{2}-\int_{s}^{t}\left\langle F-F_{0}-u E, \nabla(u-E)\right\rangle_{\Omega} d \tau
\end{aligned}
$$

for almost all $s \in(0, T)$ and all $t \in(s, T)$, and the energy estimate

$$
\begin{aligned}
\| u- & E\left\|_{2, \infty ; T^{\prime}}^{2}+\right\| \nabla(u-E) \|_{2,2 ; T^{\prime}}^{2} \\
& \leq 2 e^{\alpha T^{\prime}\|E\|_{4}^{8}}\left(\left\|u_{0}-E\right\|_{2}^{2}+2\left\|F-F_{0}\right\|_{2,2 ; T^{\prime}}^{2}+4 T^{\prime}\|E\|_{4}^{4}\right)
\end{aligned}
$$

for all finite $0<T^{\prime} \leq T$, where $\alpha \geq 0$ is an absolute constant.

Theorem 1.3 considers the worst and most general case in which the energies $(1 / 2)\|u-E\|_{2, \infty, T^{\prime}}^{2}$ and $\|\nabla(u-E)\|_{2,2, T^{\prime}}^{2}$ may grow exponentially in time with an exponent proportional to $\|E\|_{4}^{8}$, i.e., when $\alpha>0$. Here, we may simply take $E$ as the solution of the Stokes system (1.8) with data $g$ and $f_{0}=0$. However, a modification of its proof will show that under certain assumptions on the boundary data and a careful choice of $E$, better to say, of $F_{0}$ in (1.8), the exponent $\alpha$ in (1.15) may vanish. Since generally the vector field $E$ will not be the limit of the weak solution $u(t)$ as $t \rightarrow \infty$, the energy term $\|\nabla(u-E)\|_{2,2, T^{\prime}}^{2}$ necessarily implies a linear growth in time in the energy estimate.

Corollary 1.4. Let $\Omega \subset \boldsymbol{R}^{3}$ be a bounded and possibly multiply connected domain with boundary components $\Gamma_{0}, \ldots, \Gamma_{m}$ of class $C^{1,1}$. Further let $u_{0} \in$ $L^{2}(\Omega), f=\operatorname{div} F, F \in L^{2}\left(0, T ; L^{2}(\Omega)\right)$ and $g \in W^{1 / 2,2}(\partial \Omega)$ satisfy

$$
\operatorname{div} u_{0}=0,\left.\quad N \cdot u_{0}\right|_{\partial \Omega}=N \cdot g
$$

and the flux condition

$$
\int_{\Gamma_{j}} N \cdot g d \sigma=0 \quad \text { for } j=0, \ldots, m .
$$


Then there exists a vector field $E \in W^{1,2}(\Omega)$ and a weak (Leray-Hopf) solution $u$ of the Navier-Stokes system (1.1) satisfying (1.11)-(1.13) with $v=u-E$, and the energy estimate

$$
\begin{aligned}
\| u- & E\left\|_{2, \infty ; T^{\prime}}^{2}+\right\| \nabla(u-E) \|_{2,2 ; T^{\prime}}^{2} \\
& \leq 2\left\|u_{0}-E\right\|_{2}^{2}+8\|F\|_{2,2 ; T^{\prime}}^{2}+c T^{\prime}\left(\|g\|_{W^{1 / 2,2}(\partial \Omega)}^{2}+\|g\|_{W^{1 / 2,2}(\partial \Omega)}^{4}\right)
\end{aligned}
$$

for all finite $0<T^{\prime} \leq T$ where $c=c(\Omega)>0$. In particular, this result holds in a simply connected domain.

Before proving Theorem 1.3 and Corollary 1.4 in Section 2 we summarize some well-known results and introduce further notations.

For a bounded smooth domain $\Omega \subset \boldsymbol{R}^{3}$ as in Section 1 let $P: L^{2}(\Omega) \rightarrow$ $L_{\sigma}^{2}(\Omega)$ denote the Helmholtz projection, and let $A: \mathscr{D}(A) \rightarrow L_{\sigma}^{2}(\Omega), A=-P \Delta$, denote the Stokes operator with domain $D(A)=W^{2,2}(\Omega) \cap W_{0}^{1,2}(\Omega) \cap L_{\sigma}^{2}(\Omega)$ and range $\mathscr{R}(A)=L_{\sigma}^{2}(\Omega)$. Then $A^{\alpha}: \mathscr{D}\left(A^{\alpha}\right) \rightarrow L_{\sigma}^{2}(\Omega),-1 \leq \alpha \leq 1$, denote the fractional powers of $A$; it holds

$$
D(A) \subseteq D\left(A^{\alpha}\right) \subseteq L_{\sigma}^{2}(\Omega), \quad \mathscr{R}\left(A^{\alpha}\right)=L_{\sigma}^{2}(\Omega) \quad \text { for } 0 \leq \alpha \leq 1,
$$

and $\left(A^{\alpha}\right)^{-1}=A^{-\alpha}$ for $-1 \leq \alpha \leq 1$. In particular, the square root $A^{1 / 2}$ of $A$ satisfies

$$
\begin{aligned}
\left\langle A^{1 / 2} v, A^{1 / 2} w\right\rangle_{\Omega} & =\langle\nabla v, \nabla w\rangle_{\Omega} \quad \text { for } v, w \in \mathscr{D}\left(A^{1 / 2}\right) \\
& =W_{0, \sigma}^{1,2}(\Omega):=W_{0}^{1,2}(\Omega) \cap L_{\sigma}^{2}(\Omega) .
\end{aligned}
$$

Moreover, $A^{\alpha}$ is a selfadjoint operator in $L_{\sigma}^{2}(\Omega)$ for every $\alpha \in[-1,1]$.

Let $v \in \mathscr{D}\left(A^{\alpha}\right), \quad 0 \leq \alpha \leq 1 / 2$, and let $2 \leq q<\infty$ satisfy $2 \alpha+3 / q=3 / 2$. Then we obtain the embedding estimate

$$
\|v\|_{q} \leq c\left\|A^{\alpha} v\right\|_{2}
$$

with $c=c(\alpha)>0$. Moreover, if $v \in \mathscr{D}(A)$ and $0 \leq \alpha \leq 1$, then

$$
\left\|A^{\alpha} v\right\|_{2} \leq\|A v\|_{2}^{\alpha}\|v\|_{2}^{1-\alpha}
$$

and, if $v \in \mathscr{D}\left(A^{1 / 2}\right), 0 \leq \alpha \leq 1$, then

$$
\left\|A^{\alpha / 2} v\right\|_{2} \leq\left\|A^{1 / 2} v\right\|_{2}^{\alpha}\|v\|_{2}^{1-\alpha}
$$

where $\left\|A^{1 / 2} v\right\|_{2}=\|\nabla v\|_{2}$. As consequence of (1.18) and (1.20) we see that for $2 \leq q<\infty$ and $\beta=3 / 2-3 / q$ there exists a constant $c=c(\beta)>0$ such that

$$
\|v\|_{q} \leq c\|\nabla v\|_{2}^{\beta}\|v\|_{2}^{1-\beta} \quad \text { for } v \in W_{0, \sigma}^{1,2}(\Omega) .
$$


Let $F=\left(F_{i j}\right)_{i, j=1}^{3} \in L^{2}(\Omega)$ be given. Then the term $A^{-1 / 2} P \operatorname{div} F$ is welldefined (in a generalized sense) in $L_{\sigma}^{2}(\Omega)$ by the relation

$$
\left\langle A^{-1 / 2} P \operatorname{div} F, v\right\rangle_{\Omega}=\left\langle F, \nabla A^{-1 / 2} v\right\rangle_{\Omega}, \quad v \in L_{\sigma}^{2}(\Omega),
$$

and it holds

$$
\left\|A^{-1 / 2} P \operatorname{div} F\right\|_{2} \leq\|F\|_{2}
$$

indeed, $w=A^{-1 / 2}\left(A^{-1 / 2} P \operatorname{div} F\right)$ is the weak solution of the Stokes system $-\Delta w+\nabla h=\operatorname{div} F$ in $W_{0, \sigma}^{1,2}(\Omega)$ with pressure $h$. For the latter results we refer to $[16$, Chapter III].

The Yosida approximation, based on the operator $A^{1 / 2}$, is defined by the sequence of operators

$$
J_{k}=\left(I+\frac{1}{k} A^{1 / 2}\right)^{-1}, \quad k \in N
$$

where $I$ denotes the identity. As is well-known,

$$
\left\|J_{k} v\right\|_{2} \leq\|v\|_{2}, \quad\left\|\frac{1}{k} A^{1 / 2} J_{k} v\right\|_{2} \leq\|v\|_{2} \quad \text { for } k \in N, v \in L_{\sigma}^{2}(\Omega),
$$

and $J_{k} v \rightarrow v$ in $L^{2}(\Omega)$ as $k \rightarrow \infty$ for every $v \in L_{\sigma}^{2}(\Omega)$. Moreover, for $k \in N$,

$$
\left\|\nabla J_{k} v\right\|_{2}=\left\|A^{1 / 2} J_{k} v\right\|_{2}=\left\|J_{k} A^{1 / 2} v\right\|_{2} \leq\left\|A^{1 / 2} v\right\|_{2}=\|\nabla v\|_{2}, \quad v \in D\left(A^{1 / 2}\right) .
$$

The operator $-A$ generates a bounded analytic semigroup $e^{-t A}: L_{\sigma}^{2}(\Omega) \rightarrow$ $L_{\sigma}^{2}(\Omega), 0 \leq t<\infty$, such that for $0 \leq \alpha \leq 1$

$$
\left\|A^{\alpha} e^{-t A} v\right\|_{2} \leq t^{-\alpha}\|v\|_{2} \quad \text { for } t>0, v \in L_{\sigma}^{2}(\Omega)
$$

see [16, Chapter IV.1]

Finally we need some properties of the linear instationary Stokes system

$$
\begin{array}{rlrl}
v_{t}-\Delta v+\nabla h & =\hat{f}, \quad \operatorname{div} v & =0 \text { in } \Omega \times(0, T) \\
\left.v\right|_{\partial \Omega} & =0, & v(0) & =v_{0} \text { at } t=0
\end{array}
$$

with data $v_{0} \in L_{\sigma}^{2}(\Omega), \hat{f}=\operatorname{div} \hat{F}$, where $\hat{F} \in L^{2}\left(0, T ; L^{2}(\Omega)\right)$, and pressure $h$. There exists a unique weak solution $u \in L^{\infty}\left(0, T ; L_{\sigma}^{2}(\Omega)\right) \cap L^{2}\left(0, T ; W_{0}^{1,2}(\Omega)\right)$ defined by the relation

$$
-\left\langle v, w_{t}\right\rangle_{\Omega, T}+\langle\nabla v, \nabla w\rangle_{\Omega, T}=\left\langle v_{0}, w(0)\right\rangle-\langle\hat{F}, \nabla w\rangle_{\Omega, T},
$$

$w \in C_{0}^{\infty}\left([0, T) ; C_{0, \sigma}^{\infty}(\Omega)\right)$, which has the well-defined integral representation

$$
v(t)=e^{-t A} v_{0}+\int_{0}^{t} A^{1 / 2} e^{-A(t-\tau)} A^{-1 / 2} P \operatorname{div} \hat{F} d \tau, \quad 0 \leq t<T .
$$


Moreover, the solution $v$ satisfies the energy equality

$$
\frac{1}{2}\|v(t)\|_{2}^{2}+\int_{0}^{t}\|\nabla v\|_{2}^{2} d \tau=\frac{1}{2}\left\|v_{0}\right\|_{2}^{2}-\int_{0}^{t}\langle\hat{F}, \nabla v\rangle_{\Omega} d \tau, \quad 0 \leq t<T,
$$

and consequently the energy estimate

$$
\|v\|_{2, \infty ; T}^{2}+\|\nabla v\|_{2,2 ; T}^{2} \leq\left\|v_{0}\right\|_{2}^{2}+\|\hat{F}\|_{2,2 ; T}^{2} .
$$

Further, $v:[0, T) \rightarrow L_{\sigma}^{2}(\Omega)$ is strongly continuous, see [16, Chapter IV].

\section{Proofs}

There are several proofs of Theorem 1.3 when $\left.u\right|_{\partial \Omega}=g=0$, see e.g. $[11,13$, $14,16,17]$. Usually, in a first step, a sequence of approximate equations yields approximate solutions $u_{k}, k \in N$. In a second step energy estimates for $u_{k}$ with a bound independent of $k \in N$ are derived. Hence a subsequence of $\left(u_{k}\right)$ will converge in a weak sense to an element $u$ which is shown to be a solution of the original problem. One possibility is to use the Yosida approximation yielding the approximate system

$$
\begin{aligned}
u_{t}-\Delta u+\left(J_{k} u\right) \cdot \nabla u+\nabla p & =f, & & \operatorname{div} u=0 \\
\left.u\right|_{\partial \Omega} & =0, & & u(0)=u_{0} .
\end{aligned}
$$

In the following we will use a modification of this procedure and consider the approximate system

$$
\begin{aligned}
v_{t}-\Delta v+\left(J_{k} v+E\right) \cdot \nabla(v+E)+\nabla p & =f, & & \operatorname{div} v=0 \\
\left.v\right|_{\partial \Omega} & =0, & v(0) & =v_{0}
\end{aligned}
$$

where $u_{0}, E$ are given as in Definition 1.2, and

$$
\begin{array}{ll}
v_{0}=u_{0}-E \in L_{\sigma}^{2}(\Omega), & f=\operatorname{div}\left(F-F_{0}\right), \\
F \in L^{2}\left(0, T ; L^{2}(\Omega)\right), & F_{0} \in L^{2}(\Omega) .
\end{array}
$$

Then we are looking for a weak Leray-Hopf solution

$$
v=v_{k} \in L_{\mathrm{loc}}^{\infty}\left([0, T) ; L_{\sigma}^{2}(\Omega)\right) \cap L_{\mathrm{loc}}^{2}\left([0, T) ; W_{0}^{1,2}(\Omega)\right)
$$

of (2.1) with data (2.2) in the sense that

$$
\begin{gathered}
-\left\langle v, w_{t}\right\rangle_{\Omega, T}+\langle\nabla v, \nabla w\rangle_{\Omega, T}-\left\langle\left(J_{k} v+E\right) \cdot(v+E), \nabla w\right\rangle_{\Omega, T} \\
=\left\langle v_{0}, w(0)\right\rangle_{\Omega}-\left\langle F-F_{0}, \nabla w\right\rangle_{\Omega, T}
\end{gathered}
$$


for all $w \in C_{0}^{\infty}\left(\left[0, T ; C_{0, \sigma}^{\infty}(\Omega)\right)\right.$, and satisfying the energy inequality

$$
\frac{1}{2}\|v(t)\|_{2}^{2}+\int_{0}^{t}\|\nabla v\|_{2}^{2} d \tau \leq \frac{1}{2}\left\|v_{0}\right\|_{2}^{2}-\int_{0}^{t}\left\langle F-F_{0}-\left(J_{k} v+E\right) E, \nabla v\right\rangle_{\Omega} d \tau
$$

$0 \leq t<T$

Lemma 2.1. For every $k \in N$ there exists some $0<T_{k}=T\left(k,\left\|v_{0}\right\|_{2}\right.$, $\left.\left\|F-F_{0}\right\|_{2,2 ; T},\|E\|_{4}\right) \leq \min (1, T)$ such that the perturbed mollified Navier-Stokes system (2.1) has a unique weak solution

$$
v=v_{k} \in X_{T_{k}}:=L^{\infty}\left(0, T_{k} ; L_{\sigma}^{2}(\Omega)\right) \cap L^{2}\left(0, T_{k} ; W_{0}^{1,2}(\Omega)\right)
$$

in the sense (2.3), (2.4).

Proof. First assume that $v=v_{k} \in X_{k}:=X_{T_{k}}$ is a weak solution of (2.1) satisfying (2.3), (2.4), (2.5). To estimate its norm in the space $X_{k}$, i.e., the norm

$$
\|v\|_{X_{k}}=\|v\|_{2, \infty ; T_{k}}+\|\nabla v\|_{2,2 ; T_{k}}
$$

we have to analyze the nonlinear term $\left(J_{k} v+E\right)(v+E)$. By Hölder's inequality and the properties (1.18), (1.20), (1.23) with $\sigma=8, s=8 / 3, \beta=3 / 8$ we get when $0<T_{k} \leq 1$, that

$$
\begin{aligned}
\left\|\left(J_{k} v+E\right)(v+E)\right\|_{2,2 ; T_{k}} \leq & \left\|J_{k} v+E\right\|_{4, \sigma ; T_{k}}\|v+E\|_{4, s ; T_{k}} \\
\leq & c\left(\left\|A^{\beta} J_{k} v\right\|_{2, \sigma ; T_{k}}+\|E\|_{4, \sigma ; T_{k}}\right) \\
& \times\left(\left\|A^{\beta} v\right\|_{2, s ; T_{k}}+\|E\|_{4, s ; T_{k}}\right) \\
\leq & c\left(\left(\left\|A^{1 / 2} J_{k} v\right\|_{2}^{3 / 4}\left\|J_{k} v\right\|_{2}^{1 / 4}\right)_{\sigma ; T_{k}}+\|E\|_{4, \sigma ; T_{k}}\right) \\
& \times\left(\left(\left\|A^{1 / 2} v\right\|_{2}^{3 / 4}\|v\|_{2}^{1 / 4}\right)_{s ; T_{k}}+\|E\|_{4, s ; T_{k}}\right) \\
\leq & c\left(k^{3 / 4}\|v\|_{2 ; 8 ; T_{k}}+T_{k}^{1 / 8}\|E\|_{4}\right)\left(\|v\|_{X_{k}}+T_{k}^{1 / 8}\|E\|_{4}\right),
\end{aligned}
$$

where $c>0$ is an absolute constant. Hence we obtain the estimate

$$
\left\|\left(J_{k} v+E\right)(v+E)\right\|_{2,2 ; T_{k}} \leq c k^{3 / 4} T_{k}^{1 / 8}\left(\|v\|_{X_{k}}+\|E\|_{4}\right)^{2} .
$$

Defining the nonlinear operators

$$
F_{k}(v)=F-F_{0}-\left(J_{k} v+E\right)(v+E), \quad f_{k}(v)=\operatorname{div} F_{k}(v),
$$

we can write $(2.1)$ in the form

$$
\begin{aligned}
v_{t}-\Delta v+\nabla p & =f_{k}(v), & \operatorname{div} v & =0, \\
\left.v\right|_{\partial \Omega} & =0, & v(0) & =v_{0} .
\end{aligned}
$$


By the above estimate $(2.6) F_{k}(v) \in L^{2}\left(0, T_{k} ; L^{2}(\Omega)\right)$ so that $v$ may be considered as the weak solution of the Stokes system (2.8). Hence (1.26), (1.27) yield the representation and fixed point problem

$$
v=\mathscr{F}_{k}(v) \quad \text { in } X_{k}
$$

where

$$
\mathscr{F}_{k}(v)(t)=e^{-t A} v_{0}+\int_{0}^{t} A^{1 / 2} e^{-(t-\tau)} A^{-1 / 2} P \operatorname{div} F_{k}(v)(\tau) d \tau,
$$

$0 \leq t<T_{k}$. Moreover, by the energy estimate (1.29)

$$
\left\|\mathscr{F}_{k}(v)\right\|_{X_{k}} \leq c\left(\left\|v_{0}\right\|_{2}+\left\|F-F_{0}\right\|_{2,2 ; T_{k}}\right)+c k^{3 / 4} T_{k}^{1 / 8}\left(\|v\|_{X_{k}}+\|E\|_{4}\right)^{2},
$$

or, for short,

$$
\left\|\mathscr{F}_{k}(v)\right\|_{X_{k}}+d \leq a\left(\|v\|_{X_{k}}+d\right)^{2}+b
$$

where

$$
a=c k^{3 / 4} T_{k}^{1 / 8}, \quad b=c\left(\left\|v_{0}\right\|_{2}+\left\|F-F_{0}\right\|_{2,2 ; T}\right)+d, \quad d=\|E\|_{4},
$$

with an absolute constant $c>0$.

Up to now $v$ was a given solution of (2.1) in the sense of (2.3), (2.4). In the next step we solve the fixed point problem (2.9) in $X_{k}$ by Banach's fixed point theorem provided that $T_{k}>0$ is sufficiently small. For any $0<T_{k} \leq$ $\min (1, T)$ and $v \in X_{T_{k}}$ we know that $F_{k}(v) \in L^{2}\left(0, T_{k} ; L^{2}(\Omega)\right)$, that $\mathscr{F}_{k}(v)$ is well-defined and satisfies the estimate (2.11). For fixed $k \in N$ choose $T_{k}=$ $T\left(k,\left\|v_{0}\right\|_{2},\left\|F-F_{0}\right\|_{2,2 ; T_{k}},\|E\|_{4}\right)$ in $(0, \min (1, T))$ such that

$$
4 a b<1 .
$$

Then the quadratic equation $y=a y^{2}+b$ has a minimal positive root $y_{1}$, namely $y_{1}=2 b(1+\sqrt{1-4 a b})^{-1}$, satisfying $d \leq b<y_{1}<2 b$. Hence the closed ball $B_{k}=B=\left\{v \in X_{k}:\|v\|_{X_{k}} \leq y_{1}-d\right\}$ is not empty, and from (2.11) we conclude that $\mathscr{F}_{k}$ maps $B$ into $B$. Moreover, if $v, v^{\prime} \in B$, then

$$
\begin{aligned}
\left(\mathscr{F}_{k}(v)-\mathscr{F}_{k}\left(v^{\prime}\right)\right)(t)= & -\int_{0}^{t} A^{1 / 2} e^{-(t-\tau) A} A^{-1 / 2} P \operatorname{div}\left(\left(J_{k} v+E\right)\left(v-v^{\prime}\right)\right. \\
& \left.+\left(J_{k}\left(v-v^{\prime}\right)\right)\left(v^{\prime}+E\right)\right) d \tau,
\end{aligned}
$$

and the same arguments as used for (2.11) lead to the estimate

$$
\begin{aligned}
\left\|\mathscr{F}_{k}(v)-\mathscr{F}_{k}\left(v^{\prime}\right)\right\|_{X_{k}} & \leq a\left(\|v\|_{X_{k}}+\left\|v^{\prime}\right\|_{X_{k}}+2 d\right)\left\|v-v^{\prime}\right\|_{X_{k}} \\
& \leq 2 a y_{1}\left\|v-v^{\prime}\right\|_{X_{k}}<4 a b\left\|v-v^{\prime}\right\|_{X_{k}} .
\end{aligned}
$$


Hence $\mathscr{F}_{k}$ is a strict contraction on $B$. Now Banach's fixed point theorem yields the existence of a unique $v=v_{k} \in B$ satisfying $v=\mathscr{F}_{k}(v)$, i.e.,

$$
v(t)=e^{-t A} v_{0}+\int_{0}^{t} A^{1 / 2} e^{-(t-\tau) A} A^{1 / 2} P \operatorname{div} F_{k}(v)(\tau) d \tau, \quad 0 \leq t<T_{k} .
$$

Obviously, $v$ is the unique weak solution of the Stokes system (2.8) with data $f_{k}(v), v_{0}$; in particular, $v:\left[0, T_{k}\right) \rightarrow L_{\sigma}^{2}(\Omega)$ is strongly continuous, and the energy equality

$$
\frac{1}{2}\|v(t)\|_{2}^{2}+\int_{0}^{t}\|\nabla v\|_{2}^{2} d \tau=\frac{1}{2}\left\|v_{0}\right\|_{2}^{2}-\int_{0}^{t}\left\langle F_{k}(v), \nabla v\right\rangle d \tau
$$

holds for every $0 \leq t<T_{k}$.

Let us consider the term $\left\langle F_{k}(v), \nabla v\right\rangle_{\Omega}$ in (2.13) with $F_{k}(v)$ as in (2.7) more closely. Since by (2.6) $\left(J_{k} v+E\right)(v+E)(\tau) \in L^{2}(\Omega)$ and $\nabla v(\tau) \in L^{2}(\Omega)$ for almost all $\tau \in\left[0, T_{k}\right)$, we get for these $\tau$

$$
\begin{aligned}
\left\langle\left(J_{k} v+E\right)(v+E), \nabla v\right\rangle_{\Omega} & =\left\langle\left(J_{k} v+E\right) E, \nabla v\right\rangle_{\Omega}+\frac{1}{2} \int_{\Omega}\left(J_{k} v+E\right) \nabla\left(|v|^{2}\right) d x \\
& =\left\langle\left(J_{k} v+E\right) E, \nabla v\right\rangle_{\Omega} .
\end{aligned}
$$

This identity and (2.13) yield the energy identity

$$
\frac{1}{2}\|v(t)\|_{2}^{2}+\int_{0}^{t}\|\nabla v\|_{2}^{2} d \tau=\frac{1}{2}\left\|v_{0}\right\|_{2}^{2}-\int_{0}^{t}\left\langle F-F_{0}-\left(J_{k} v+E\right) E, \nabla v\right\rangle_{\Omega} d \tau,
$$

i.e., (2.4) with " $=$ " instead of " $\leq$ " for $t \in[0, T)$. Moreover, $v$ satisfies $(2.3)$ in $\Omega \times\left(0, T_{k}\right)$. Thus $v$ is a weak solution of $(2.1)$ with data $(2.2)$ in $\left(0, T_{k}\right) \times \Omega$.

To prove the uniqueness of this solution $v$ not only in the ball $B=$ $B_{k} \subset X_{k}$, but in the whole of $X_{k}$, let $w \in X_{k}$ be another weak solution of (2.1), (2.2) in $\Omega \times\left(0, T_{k}\right)$. Then $w=\mathscr{F}_{k}(w)$ with $\mathscr{F}_{k}$ as in (2.10), and the estimate (2.12) with $\|\cdot\|_{X_{T^{\prime}}}$ replacing $\|\cdot\|_{X_{T_{k}}}$ for any $0<T^{\prime} \leq T_{k}$ implies that

$$
\|v-w\|_{X_{T^{\prime}}}=\left\|\widetilde{\mathscr{F}}_{k}(v)-\widetilde{\mathscr{F}}_{k}(w)\right\|_{X_{T^{\prime}}} \leq a^{\prime}\left(\|v\|_{X_{T^{\prime}}}+\|w\|_{X_{T^{\prime}}}+2 d\right)\|v-w\|_{X_{T^{\prime}}}
$$
where $a^{\prime}=c k^{3 / 4}\left(T^{\prime}\right)^{1 / 8}$ and $d=\|E\|_{4}$. Now we choose

$$
T^{\prime}=T^{\prime}\left(k,\|v\|_{X_{k}},\|w\|_{X_{k}},\|E\|_{4}\right) \in\left(0, T_{k}\right)
$$

in such a way that $a^{\prime}\left(\|v\|_{X_{k}}+\|w\|_{X_{k}}+2 d\right) \leq 1 / 2$. Then we obtain from (2.14) that $v \equiv w$ in $\left[0, T^{\prime}\right)$. Repeating this procedure finitely many times with the same $T^{\prime}$ we finally get that $v=w$ in $\left[0, T_{k}\right)$. This completes the proof of Lemma 2.1 .

For the final passage to the limit $k \rightarrow \infty$ in the proof of Theorem 1.3 we need an energy estimate which holds uniformly in $k \in N$. 
Lemma 2.2. For $k \in N$ let $v=v_{k}$ be a weak solution of the perturbed mollified Navier-Stokes system (2.1) with data (2.2) in $[0, T) \times \Omega, 0<T<\infty$. Then there exists an absolute constant $\alpha \geq 0$ not depending on $k \in N$ such that the energy estimate

$$
\|v\|_{2, \infty ; T}^{2}+\|\nabla v\|_{2,2 ; T}^{2} \leq 2 e^{\alpha T\|E\|_{4}^{8}}\left(\left\|v_{0}\right\|_{2}^{2}+2\left\|F-F_{0}\right\|_{2,2 ; T}^{2}+4 T\|E\|_{4}^{4}\right)
$$

holds.

Proof. By a slight modification of the proof of (2.6) where $0<T_{k} \leq 1$ was assumed we get that $\left(J_{k} v+E\right)(v+E) \in L^{2}\left(0, T ; L^{2}(\Omega)\right)$ and consequently that

$$
F_{k}(v) \in L^{2}\left(0, T ; L^{2}(\Omega)\right),
$$

see (2.7). Moreover, $v$ is the weak solution of the Stokes system (2.8) so that $v:[0, T) \rightarrow L_{\sigma}^{2}(\Omega)$ is strongly continuous.

From the energy inequality (2.4) we obtain for each $t \in(0, T)$ the estimate

$$
\begin{aligned}
& \frac{1}{2}\|v(t)\|_{2}^{2}+\int_{0}^{t}\|\nabla v\|_{2} d \tau \\
& \quad \leq \frac{1}{2}\left\|v_{0}\right\|_{2}^{2}+\int_{0}^{t}\left(\left\|F-F_{0}\right\|_{2}+\left\|\left(J_{k} v+E\right) E\right\|_{2}\right)\|\nabla v\|_{2} d \tau .
\end{aligned}
$$

Next, by Young's inequality

$$
\int_{0}^{t}\left\|F-F_{0}\right\|_{2}\|\nabla v\|_{2}^{2} d \tau \leq \frac{1}{4} \int_{0}^{t}\|\nabla v\|_{2}^{2} d \tau+\int_{0}^{t}\left\|F-F_{0}\right\|_{2}^{2} d \tau,
$$

and by Hölder's inequality and (1.21)

$$
\begin{aligned}
\int_{0}^{t}\left\|\left(J_{k} v+E\right) E\right\|_{2}\|\nabla v\|_{2} d \tau \leq & \int_{0}^{t}\left(\left\|J_{k} v\right\|_{4}\|E\|_{4}+\|E\|_{4}^{2}\right)\|\nabla v\|_{2} d \tau \\
\leq & c \int_{0}^{t}\left\|\nabla\left(J_{k} v\right)\right\|_{2}^{3 / 4}\left\|J_{k} v\right\|_{2}^{1 / 4}\|E\|_{4}\|\nabla v\|_{2} d \tau \\
& +\int_{0}^{t}\|E\|_{4}^{2}\|\nabla v\|_{2} d \tau .
\end{aligned}
$$

Hence, by (1.23), (1.24) and Young's inequality,

$$
\begin{aligned}
\int_{0}^{t}\left\|\left(J_{k} v+E\right) E\right\|_{2}\|\nabla v\|_{2} d \tau & \leq c \int_{0}^{t}\|\nabla v\|_{2}^{7 / 4}\|v\|_{2}^{1 / 4}\|E\|_{4} d \tau+\int_{0}^{t}\|E\|_{4}^{2}\|\nabla v\|_{2} d \tau \\
& \leq \frac{1}{4} \int_{0}^{t}\|\nabla v\|_{2}^{2} d \tau+c^{\prime} \int_{0}^{t}\|v\|_{2}^{2}\|E\|_{4}^{8} d \tau+2 t\|E\|_{4}^{4}
\end{aligned}
$$


with absolute constants $c, c^{\prime}>0$. Summarizing the previous estimates we deduce from (2.17) the inequality

$$
\|v(t)\|_{2}^{2}+\int_{0}^{t}\|\nabla v\|_{2}^{2} d \tau \leq\left\|v_{0}\right\|_{2}^{2}+2 \int_{0}^{t}\left\|F-F_{0}\right\|_{2}^{2} d \tau+4 t\|E\|_{4}^{4}+2 c^{\prime}\|E\|_{4}^{8} \int_{0}^{t}\|v\|_{2}^{2} d \tau
$$

for every $0<t<T$. Now Gronwall's Lemma yields the estimate (2.16).

Lemma 2.3. For every $k \in N$ the perturbed mollified Navier-Stokes equation system (2.1) with data (2.2) has a unique global weak solution $v_{k}$ in $[0, T) \times \Omega$, $0<T \leq \infty$, satisfying (2.3), the energy inequality (2.4) and the energy estimate (2.16) with $T$ replaced by each finite $0<T^{\prime}<T$.

Proof. By Lemmata 2.1 and 2.2 there exists a weak solution $v_{k}$ in some interval $\left[0, T_{k}\right)$ with $0<T_{k} \leq T$. Let $\left[0, T^{*}\right), T_{k} \leq T^{*} \leq T$, be the largest interval within $[0, T)$ such that a weak solution with the above properties exists. If $T^{*}=T$, then the proof is complete; the energy estimate (1.15) follows from (2.16).

Thus suppose that $0<T^{*}<T$. Then $T^{*}<\infty$ and we apply Lemma 2.2 and the energy estimate (2.16) with $T$ replaced by $T^{*}$. Since $v_{k}$ is strongly $L_{\sigma}^{2}(\Omega)$-continuous in $\left[0, T^{*}\right)$, we can choose some $T_{0} \in\left(0, T^{*}\right)$ close to $T^{*}$ such that Lemma 2.1 yields the existence of a unique weak solution of (2.1) with initial value $v_{k}\left(T_{0}\right) \in L_{\sigma}^{2}(\Omega)$ in some interval $\left[T_{0}, T_{1}\right)$ with $T_{1}<T^{*}$. Indeed, the length of the interval of existence, $T_{1}-T_{0}$, is determined by $T\left(k,\left\|v_{k}\left(T_{0}\right)\right\|_{2}\right.$, $\left.\|F\|_{2,2 ; T},\left\|F_{0}\right\|_{2},\|E\|_{4}\right)$, see Lemma 2.1 , where by $(2.16)$

$$
\left\|v_{k}\left(T_{0}\right)\right\|_{2}^{2} \leq e^{\alpha T^{*}\|E\|_{4}^{8}}\left(\left\|v_{0}\right\|_{2}^{2}+2\left\|F-F_{0}\right\|_{2,2 ; T^{*}}^{2}+4 T^{*}\|E\|_{4}^{4}\right) .
$$

Thus there exists a $\delta>0$ independent of $T_{0}$ close to $T^{*}$ such that the existence of the weak solution starting at $T_{0}$ is guaranteed on $\left[T_{0}, T_{0}+\delta\right)$.

This procedure allows to extend the given weak solution from $\left[0, T^{*}\right)$ to $\left[0, T_{1}\right) \supset\left[0, T^{*}\right]$ in contradiction to choice of $T^{*}$. The uniqueness of the extended solution $v_{k}$ follows in the same way as in the proof of Lemma 2.1.

Now we are in a position to pass to the limit $k \rightarrow \infty$ for the functions $v_{k}$ and to prove Theorem 1.3.

Proof of Theorem 1.3. First consider the case $0<T<\infty$, so that the sequence of weak solutions, $\left(v_{k}\right)$, constructed in Lemma 2.1-2.3, satisfies the energy estimate

$$
\left\|v_{k}\right\|_{2, \infty ; T}^{2}+\left\|\nabla v_{k}\right\|_{2,2 ; T}^{2} \leq c\left(\left\|v_{0}\right\|_{2}^{2}+\left\|F-F_{0}\right\|_{2,2 ; T}^{2}+\|E\|_{4}^{4}\right)
$$


with a constant $c=c\left(T,\|E\|_{4}\right)>0$, see (2.16). Then there exists

$$
v \in L^{\infty}\left(0, T ; L_{\sigma}^{2}(\Omega)\right) \cap L^{2}\left(0, T ; W_{0}^{1,2}(\Omega)\right)
$$

and a subsequence of $\left(v_{k}\right)$, which for simplicity is again denoted by $\left(v_{k}\right)$, with the following properties:

$$
\begin{array}{ll}
v_{k} \text { converges weakly to } v & \text { in } L^{2}\left(0, T ; W_{0}^{1,2}(\Omega)\right) \\
v_{k} \text { converges strongly to } v & \text { in } L^{2}\left(0, T ; L^{2}(\Omega)\right) \\
v_{k}(t) \text { converges strongly to } v(t) & \text { in } L_{\sigma}^{2}(\Omega) \text { for a.a. } t \in[0, T)
\end{array}
$$

as $k \rightarrow \infty$. For the proof of the crucial result $(2.20)_{2}$ we refer to [16, V. 3.2, $3.3]$ together with some necessary modifications due to the perturbation terms.

To prove that $v$ is a weak solution of the perturbed Navier-Stokes system, we still have to show that $v$ solves the variational problem

$$
\begin{gathered}
-\left\langle v, w_{t}\right\rangle_{\Omega, T}+\langle\nabla v, \nabla w\rangle_{\Omega, T}+\langle(v+E)(v+E), \nabla w\rangle_{\Omega, T} \\
=\left\langle u_{0}-E, w(0)\right\rangle_{\Omega}-\left\langle F-F_{0}, \nabla w\right\rangle_{\Omega, T}
\end{gathered}
$$

for any test function $w \in C_{0}^{\infty}\left([0, T) ; C_{0, \sigma}^{\infty}(\Omega)\right)$. Due to $(2.20),(1.23)$

$$
\begin{aligned}
\left\langle v_{k}, w_{t}\right\rangle_{\Omega, T} & \rightarrow\left\langle v, w_{t}\right\rangle_{\Omega, T}, \\
\left\langle\nabla v_{k}, \nabla w\right\rangle_{\Omega, T} & \rightarrow\langle\nabla v, \nabla w\rangle_{\Omega, T}, \\
\left\langle\left(J_{k} v_{k}+E\right)\left(v_{k}+E\right), \nabla w\right\rangle_{\Omega, T} & \rightarrow\langle(v+E)(v+E), \nabla w\rangle_{\Omega, T}, \\
\left\langle\left(J_{k} v_{k}+E\right) E, \nabla v_{k}\right\rangle_{\Omega, T} & \rightarrow\langle(v+E) E, \nabla v\rangle_{\Omega, T}, \quad 0 \leq t<T,
\end{aligned}
$$

as $k \rightarrow \infty$; for the fourth property we also need (1.21) and Lebesgue's theorem on dominated convergence to get that $\int_{0}^{t}\left\|J_{k} v_{k}-v\right\|_{4}^{2} d \tau \rightarrow 0$. Hence $v=u-E$ is a solution of $(2.21)$ and $v(0)=v_{0}$.

Further we obtain that

$$
\|\nabla v\|_{2,2 ; T} \leq \liminf _{k \rightarrow \infty}\left\|\nabla v_{k}\right\|_{2,2 ; T}
$$

Now the energy identity (2.14) for $v_{k}$ yields with the help of $(2.20)_{4}$ for almost all $s \in(0, T)$ including $s=0$ the strong energy inequality

$$
\frac{1}{2}\|v(t)\|_{2}^{2}+\int_{s}^{t}\|\nabla v\|_{2}^{2} d \tau \leq \frac{1}{2}\|v(s)\|_{2}^{2}-\int_{s}^{t}\left\langle F-F_{0}-(v+E) E, \nabla v\right\rangle_{\Omega} d \tau .
$$


First, this inequality is proved only for a.a. $t \in(0, T)$, cf. $(2.20)_{3}$; however, since $v$ is weakly $L^{2}$-continuous, the estimate holds for all $t \in(0, T)$. Since $v=u-E$ satisfies (2.19) and $E \in W^{1,2}(\Omega)$, we also get that $u$ satisfies (1.4). This proves Theorem 1.3 when $0<T<\infty$.

Now let $T=\infty$. This case can be reduced to the situation above by using the method of diagonal sequences. Choose an strictly increasing sequence $\left(T_{j}\right)_{j=1}^{\infty}$ with $\lim _{j \rightarrow \infty} T_{j}=\infty$ and let $\left(v_{k}\right)$ denote a sequence of approximate solutions in $[0, \infty) \times \Omega$ as in Lemma 2.1-2.3. For $T=T_{1}$ we find a subsequence $\left(v_{k}^{(1)}\right)$ of $\left(v_{k}\right)$ with the properties $(2.14),(2.16),(2.18)-(2.20)$ and obtain a solution $v^{(1)}$ in $\left[0, T_{1}\right) \times \Omega$. Then we choose a subsequence $\left(v_{k}^{(2)}\right)$ of $\left(v_{k}^{(1)}\right)$ to obtain a solution $v^{(2)}$ in $\left[0, T_{2}\right) \times \Omega$ such that $\left.v^{(2)}\right|_{\left[0, T_{1}\right]}=v^{(1)}$. Proceeding in this way and finally choosing the diagonal sequence of the sequences $\left(v_{k}^{(j)}\right)$, $j \in N$, we get by passing to the limit a global in time weak solution of the perturbed Navier-Stokes system $v$, such that $\left.v\right|_{\left[0, T_{j}\right)}=v^{(j)}, j \in \boldsymbol{N}$. Obviously $v=u-E$ satisfies the energy inequality (1.13) and the energy estimate (1.15) in $[0, \infty)$.

Now the proof of Theorem 1.3 is complete.

Proof of Corollary 1.4. Let the boundary data $g \in W^{1 / 2,2}(\partial \Omega)$ satisfy the flux condition (1.16). Then a classical result [9, Lemma VIII. 4.2] yields for any $\varepsilon>0$ a vector field $E_{\varepsilon} \in W^{1,2}(\Omega)$ such that $\operatorname{div} E_{\varepsilon}=0$ and $\left.E_{\varepsilon}\right|_{\partial \Omega}=g$, and the trilinear form

$$
b(u, v, w)=\int_{\Omega} u \cdot \nabla v \cdot w d x, \quad u, v, w \in W^{1,2}(\Omega),
$$

satisfies the estimate

$$
\left|b\left(v, E_{\varepsilon}, v\right)\right| \leq \varepsilon\|\nabla v\|_{2}^{2}, \quad v \in W_{0}^{1,2}(\Omega)
$$

see also [12] for the construction of $E_{\varepsilon}$ and applications.

To apply Theorem 1.3 let $F_{0}:=-\nabla E_{\varepsilon} \in L^{2}(\Omega)$ and note that by $[9$, Lemma VIII. 4.2]

$$
\left\|F_{0}\right\|_{2} \leq\left\|E_{\varepsilon}\right\|_{W^{1,2}(\Omega)} \leq c\|g\|_{W^{1 / 2,2}(\partial \Omega)}
$$

with a constant $c=c(\varepsilon, \Omega)>0$.

We use the strong energy inequality (1.14) with $s=0$ for $v=u-E$, $E=E_{\varepsilon}$, in the form

$$
\frac{1}{2}\|v(t)\|_{2}^{2}+\int_{0}^{t}\|\nabla v\|_{2}^{2} d \tau \leq \frac{1}{2}\|v(0)\|_{2}^{2}-\int_{0}^{t}\left\langle F-F_{0}-E E, \nabla v\right\rangle_{\Omega} d \tau+\int_{0}^{t}\langle v E, \nabla v\rangle_{\Omega} d \tau
$$


and apply the estimate of the trilinear form to the last term with $\varepsilon=1 / 4$. Then we get for any $0<t<T$ the inequality

$$
\begin{aligned}
\frac{1}{2}\|v(t)\|_{2}^{2}+\int_{0}^{t}\|\nabla v\|_{2}^{2} d \tau \leq & \frac{1}{2}\left\|v_{0}\right\|_{2}^{2}+\frac{1}{4} \int_{0}^{t}\|\nabla v\|_{2}^{2} d \tau \\
& +\int_{0}^{t}\left(\left\|F-F_{0}\right\|_{2}+\|E\|_{4}^{2}\right)\|\nabla v\|_{2} d \tau .
\end{aligned}
$$

A further application of Young's inequality and the above estimate of $F_{0}$ imply (1.17).

\section{References}

[1] Amann, H., On the strong solvability of the Navier-Stokes equations, J. Math. Fluid Mech., 2 (2000), 16-98.

[2] Amann, H., Navier-Stokes equations with nonhomogeneous Dirichlet data, J. Nonlinear Math. Phys., 10 (2003), Suppl. 1, 1-11.

[ 3 ] Farwig, R., Galdi, G. P., and Sohr, H., A new class of weak solutions of the Navier-Stokes equations with nonhomogeneous data, J. Math. Fluid Mech., 8 (2006), 423-444.

[4] Farwig, R., Kozono, H., and Sohr, H., Very weak, weak and strong solutions to the instationary Navier-Stokes system, Topics on partial differential equations, ed. by $\mathrm{P}$. Kaplický, Š. Nečasová. J. Nečas Center Math. Model., Lecture Notes, Praha, 2 (2007), $1-54$.

[ 5] Farwig, R., Kozono, H., and Sohr, H., Global Leray-Hopf weak wolutions of the NavierStokes equations with nonzero time-dependent boundary values, Manuscript 2010.

[6] Farwig, R., Sohr, H., and Varnhorn, W., On optimal initial value conditions for local strong solutions of the Navier-Stokes equations, Ann. Univ. Ferrara, Sez. VII, 55 (2009), 89-110.

[ 7 ] Fursikov, A. V., Gunzburger, M. D., and Hou, L. S., Inhomogeneous boundary value problems for the three-dimensional evolutionary Navier-Stokes equations, J. Math. Fluid Mech., 4 (2002), 45-75.

[8] Galdi, G. P., An Introduction to the Mathematical Theory of the Navier-Stokes Equations, Vol. I, Springer Verlag, New York, 1994.

[9] Galdi, G. P., An Introduction to the Mathematical Theory of the Navier-Stokes Equations, Vol. II, Springer Verlag, New York, 1994.

[10] Grubb, G., Nonhomogeneous Dirichlet Navier-Stokes problems in low regularity $L_{p}$ Sobolev spaces, J. Math. Fluid Mech., 3 (2001), 57-81.

[11] Heywood, J. G., The Navier-Stokes Equations: On the existence, regularity and decay of solutions, Indiana Univ. Math. J., 29 (1980), 639-681.

[12] Hopf, E., Über die Anfangswertaufgabe für die hydrodynamischen Grundgleichungen, Math. Nachr., 4 (1950), 213-231.

[13] Ladyzhenskaya, O. A., The Mathematical Theory of Viscous Incompressible Flow, Gordon and Breach, New York, 1969.

[14] Leray, J., Sur le mouvement d'un liquide visqueux emplissant l'espace, Acta Math., 63 (1934), 193-248.

[15] Raymond, J. P., Stokes and Navier-Stokes equations with nonhomogeneous boundary conditions, Ann. Inst. H. Poincaré, Anal. Non Linéaire, 24 (2007), 921-951. 
[16] Sohr, H., The Navier-Stokes Equations, Birkhäuser Verlag, Basel, 2001.

[17] Solonnikov, V. A., Estimates for solutions of nonstationary Navier-Stokes equations, J. Soviet Math., 8 (1977), 467-529.

\author{
nuna adreso: \\ R. Farwig \\ Fachbereich Mathematik \\ Technische Universität Darmstadt \\ 64283 Darmstadt \\ Germany \\ E-mail: farwig@mathematik.tu-darmstadt.de \\ H. Kozono \\ Mathematical Institute \\ Tôhoku University \\ Sendai 980-8578 \\ Japan \\ E-mail: kozono@math.tohoku.ac.jp \\ H. Sohr \\ Fakultät für Elektrotechnik \\ Informatik und Mathematik \\ Universität Paderborn \\ 33098 Paderborn \\ Germany \\ E-mail: hsohr@math.uni-paderborn.de
}

(Ricevita la 22-an de decembro, 2009) 